\title{
The Stochastic Bidding Strategy of Micro grid for Participation in Energy and Ancillary Services
}

\author{
Iman Narimani ${ }^{1}$, Saed Reza Goldani ${ }^{2}$ \\ ${ }^{1}$ Member of Young elite sponsors institution (nokhbeha.com), Tehran, Iran ${ }^{1}$ \\ ${ }^{2}$ Assistant Professor- Faculty of Electrical and Computer Engineering, University of Birjand, \\ Birjand, Iran ${ }^{2}$
}

\begin{abstract}
Typically, micro-grid is considered as an effective way to integration of distributed generations. However, in the deregulated environment integration of micro-grid in power system should be further considered. In this paper, a stochastic bidding strategy of joint market for the energy and spinning reserve services markets, with taking into account of the uncertainties of renewable distributed generation output power, load and electricity market prices to maximize the profits of micro-grid, is recommended. The bidding strategy is modeled as an optimization problem and is divided into two stages. First the predictions for uncertainties was carried out by using Adaptive Neural Fuzzy Inference System(ANFIS) and according to the forecast errors and Latin Hyperbolic Sampling (LHS) method, scenarios for micro-grid produced and these scenarios is reduced by using backward scenario reducing. In the second stage, according to the generated scenarios in the previous stage, the profit of micro-grid bidding including the revenue of micro-grid bidding, operation and imbalance costs is optimized by genetic algorithm. In the end, micro-grid bids is sent to market. The effectiveness of the proposed method is discussed on a sample micro-grid and results show the effectiveness of the proposed method.
\end{abstract}

Keywords: Bidding strategy, Uncertainty, Power market, Ancillary services, Spinning reserve, Micro-grid, Distributed generation.

\section{INTRODUCTION}

In recent decades, we have witnessed the increasing influence of Distributed Generations (DGs) in power systems. Integration of DG units with network is one of the interesting options for responding to the ever-increasing load demand and improvement of reliability. Furthermore, the ability to provide ancillary services, such as spinning reserve,

\footnotetext{
${ }^{1}$ Electronic address : narimani.iman@yahoo.com

${ }^{2}$ Electronic address : sgoldani@birjand.ac.ir
} 
non-spinning reserve, and reactive power injection and etc. by the DGs close to the loads, is considered as an efficient and economical solution for supporting the system. Therefore it is expected that in deregulated environment, in addition to supply the future energy needs, DGs due to their ability in providing ancillary services, gain a considerable significance. But in this regard, there are some problems that cause the presence of these units in the electricity market for providing ancillary services to be challenged.

Renewable DG units due to free and green energy resources are widely used in power systems. However their uncertain nature, limited predictability of generated electricity from these units and the need to create Spot market for maintaining the network stability made the presence of these resources dependent on paying remarkable deviation penalties in the process of adjustment and settlement of the market.

On the other hand, the current policy of installing DGs has been focused on connection rather than integration. Clearly, under this regime, DGs are not visible to the system furthermore they lack the required conditions for providing support and security for the system. It seems that under such conditions, DGs cannot be considered as a reliable option to be presented in electricity market and provide critical services like ancillary services.

To solve these problems, DG should be integrated with the system operation. This goal can be achieved by integration of distributed generation or more accurately, by distributing energy resources (DER) in a micro-grid.

Even though micro-grid has many benefits, but the integration of micro-grids into the traditional distribution system imposes technical challenges for system operation in several aspects, such as energy management strategy, protection design, reliability and so on. Among the above mentioned issues, realization of interaction paradigm between micro-grid and traditional system for maximizing potential benefits of micro-grid is so crucial.

References [2] to [5] examine the participation of micro-grid in ancillary services and energy markets. These researches facilitate the effectiveness and profitability of micro-grids. However, uncertainties including renewable DG units' power output, market price, and lines/units reliability are not considered in these references. In reference [6], some of uncertainties including power output of renewable DG units and load is taken into account. Furthermore, high accuracy will be attainable when the time horizon of study is short-term, therefore in [7] the Intra-day Market (IM) is suggested for reducing the impact of uncertainty. In this method, during the day light, in several periods of time, the micro-grid will be allowed to update its suggestions that have offered to the market. 
Given that the price fluctuations in electricity market can be the main factor of profit maximization of the market players, and because this issue is not considered in the market so far, in this paper, a stochastic bidding strategy to participation of micro-grid day ahead market is proposed. This strategy will take into account the uncertainties such as the output power of renewable DG units, load and fluctuations in the market price.

The proposed method is executed on a small sample network which consists of several units of renewable distributed generation, fuel cells, micro turbines and interruptible load. The results are compared with Methods that does not take into account any kind of uncertainty. The simulation results showed the effectiveness of the proposed method. This method can also increase micro-grid profit ability in the electricity market.

\section{DEFITITIONS AND ASSUMPTIONS}

\subsection{Components of micro-grid}

The micro-grid under study consists of DG units (including dispatchable and nondispatchable units) and final consumers (including interruptible and non- interruptible loads). The ownership and management of All of DG units is undertaken by micro-grid. The energy of final consumers is provided at Retail price. This price has been set in several levels for different periods of time in a day. The micro-grid also signs an agreement about maximum amount of load interruption $\left(P_{\text {curt }}^{\max }\right)$, interruption hours and costs of interruption $\left(\mathbf{C}\left(P_{\text {curt }}\right)\right)$ with interruptible loads.

\subsection{Modeling of micro-grid components}

Modeling for the sake of participation in electricity market means that power production costs can be determined by micro-grid components. In studies of participation in electricity market, the time horizon is usually short-term and only operating costs of power generation is modeled. The operating cost of Dispatchable DG units is modeled as a quadratic function of their productivity, which is as the follow [5]:

$$
\mathbf{C}\left(P_{D G}\right)=\alpha_{i}+\beta_{i} \mathbf{P}_{D G}+\gamma_{i} \mathbf{P}_{D G}^{2}
$$

Where $\alpha_{i}, \beta_{i}$ and $\gamma_{i}$ are Positive Coefficients of the cost function. 
Operating costs of non-dispatchable distributed generation units is limited to maintenance costs, so they can be ignored.

Based on what is in [3], the consumer's cost curve of a load to cut (limit) it's load, is assumed as a function of un-served load and is modeled as a quadratic polynomial.

$$
\mathbf{C}\left(P_{\text {curt }}\right)=\alpha_{\text {int }} \mathbf{P}_{\text {curt }}+\beta_{\text {int }} \mathbf{P}_{\text {curt }}^{2}
$$

Where $\alpha_{i n t}$ and $\beta_{\text {int }}$ are not used load Coefficients.

\subsection{Market framework}

The aim of this study is to maximize the benefit of the micro-grid presence in the energy and ancillary services markets. Therefore market framework that is taken into consideration for this study is a common model for both energy and ancillary services (here spinning reserve) days markets. The market framework is shown in Figure 1. According to the forecasted and received information, the central controller sends energy and spinning reserve bids 24 hours of next day (at 12 to 36 hour time horizons) to the market operator. Power and price can be included in the bids. However, it has been assumed that the micro-grid is a price taker.

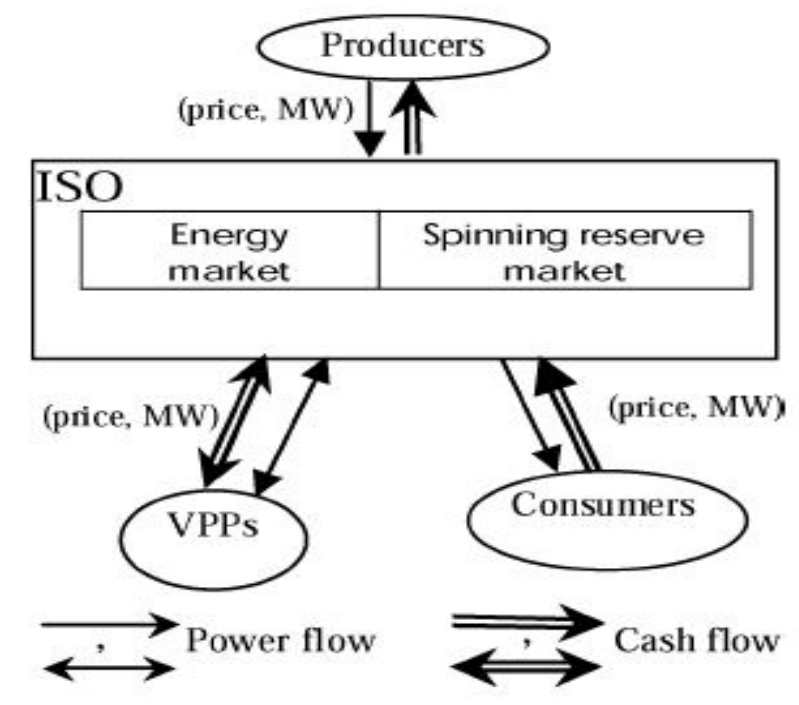

Fig. 1: Market framework

\section{BIDIING STRATEGY OF MICRO-GRID IN DAY AHEAD MARKET}

The bidding strategy contains information about market participants willing to buy or sell energy as well as some information about how much power, at what price, in what area and at what time must be exchanged. And it has very significance role in maximizing the 
profit of market participants. Generally, bidding strategies can be divided into two categories: equilibrium and non-equilibrium strategies.

Equilibrium models such as supply function and Cournot equilibrium are widely applied for the development of the bidding strategy of generation companies (GenCos) and analyzing market power in energy markets. However, unit constraints such as minimum on/off time, ramping limits, and startup and shut down costs are not considered in most of the equilibrium models.

Price-based unit commitment (PBUC) non-equilibrium model can overcome these constraints and is a good strategy for the multiple markets (energy and ancillary services). It is worth to mention that there are noticeable differences between bidding strategy of a traditional Genco and a micro-grid based on PBUC. These differences are as follows:

- A traditional Genco is only a producer; however, a micro-grid may be an entity with dual role including producer and consumer based on the direction of exchanged power with upstream grid.

- In bidding strategy of a traditional Genco, because of using non-equilibrium models based on the forecasting market prices, the supply-demand balancing constraint does not exist; but, it is a critical constraint for micro-grids.

- Unlike the traditional Genco, the DER that belongs to micro-grid may be connected to various points in distribution network; therefore the network characteristics (network topology, impedances, losses, and constraints) may impact the proposals making of micro-grids.

Micro-grid, based on the condition and capacity of distributed energy resources of its components, Consumption Load prediction and forecasting of energy and Ancillary Services prices in the electricity market attempted to plan and send its bids to the energy and Ancillary Services. With regard to the conditions and criteria of micro-grid units, bidding strategy of micro-grid must be a PBUC.

\subsection{Objective function}

The aim of the micro-grid for participating in power and ancillary services markets is to maximize the profit by providing consuming load of its consumer, power exchange with the upstream network, electricity market and the provision of ancillary services. The objective function of the problem is given in (3). The first term of objective function represents the total 
revenue from bidding in market and providing power to end customers. The Second term is unit cost to provide power and ancillary services, the third term is for payback cost of load interruption and the fourth term is imbalances costs.

$$
\begin{aligned}
\text { profit }= & \sum_{t=1}^{24}\left(\rho_{E, t} \times \mathbf{E}_{t}+\rho_{A S, t} \times \mathbf{A} \mathbf{S}_{t}+\rho_{L, t} \times \mathbf{L}_{t}\right)- \\
& \sum_{t=1}^{24}\left(\sum_{i=1}^{N_{D G}}\left(\mathbf{C}_{D G i, t}\left(\mathbf{E}_{D G i, t}+\mathbf{A} \mathbf{S}_{D G i, t}\right)\right)\right)- \\
& \sum_{t=1}^{24}\left(\sum_{k=1}^{N_{\text {curt }}}\left(\mathbf{C}_{\text {curt } k, t} \times \mathbf{A} \mathbf{S}_{\text {curt } k, t}\right)\right)-\sum_{t=1}^{24} \mathbf{C}_{\Delta p, t}
\end{aligned}
$$

Where: $\rho$ is price in energy and ancillary services markets and retain rate. $N_{D G}, N_{\text {curt }}, \mathrm{C}$ and AS are the number of dispatchable DG units, the number of interruptible loads, cost function, energy bid for energy market and energy bid for ancillary services market, respectively.

The cost of imbalances are calculated as in (4).

$$
\mathbf{C}_{\triangle p}=\left\{\begin{array}{ccc}
\rho_{u p}\left(\mathbf{P}_{G E N}-\mathbf{P}_{D M}\right) & \text { if } & P_{G E N} \geq P_{D M} \\
\rho_{\text {down }}\left(\mathbf{P}_{D M}-\mathbf{P}_{G E N}\right) & \text { if } & P_{G E N} \leq P_{D M}
\end{array}\right.
$$

Where: $\mathbf{P}_{D M}$ is the power bid for the market, $\mathbf{P}_{G E N}$ is the real time generation, $\rho_{u p}$ and down $\rho_{\text {down }}$ are price in negative and positive markets respectively.

\subsection{Constraints}

As it is mentioned before, In addition to Constraints of DG, the Constraints of intranetwork of micro-grid and balance of supply and demand should be considered.

Constraint related to the balance of supply and demand for energy and ancillary services is given in (5).

$$
\mathbf{E}_{t}+\sum_{i=1}^{N_{D G}}\left(E_{D G i, t}+A S_{D G i,}\right)+\sum_{k=1}^{N_{\text {curt }}} A S_{\text {curt } k, t}=\mathbf{L}_{t}+\text { Loss. }
$$

Where $\mathrm{L}$ is load of micro-grid and LOSS is losses of micro-grid.

Constraint related to the DER are as follow:

- DG power generation limits

$$
P_{D G i, t}^{\min } \leq\left(P_{D G i, t}+A S_{D G i, t}\right) \times I_{i, t} \leq P_{D G i, t}^{\max } .
$$


Where I is binary variable of DG situation.

- Power generation decrease and increase rate:

$$
\left\{\begin{array}{l}
P_{D G i, t+1}-P_{D G i, t} \geq R_{D G u i, t} \\
P_{D G i, t}-P_{D G i, t+1} \geq R_{D G d i, t}
\end{array}\right. \text {. }
$$

Where $R_{D G u}$ and $R_{D G d}$ are Power generation increase and decrease rate, respectively.

- Minimum on and off time:

$$
\left\{\begin{array}{l}
{\left[T_{i, t-1}^{o f f}-M D T_{i}\right] \times\left[I_{i, t-1}-I_{i, t}\right] \geq 0} \\
{\left[T_{i, t-1}^{o n}-M U T_{i}\right] \times\left[I_{i, t-1}-I_{i, t}\right] \geq 0}
\end{array} .\right.
$$

Where $T^{\text {off }}$ is number of DG's off time, $T^{\text {on }}$ number of DG's on time, MUT minimum hour of on time, MDT minimum hour of off time.

- Maximum provision power for spinning reserve:

$$
P_{D G i, t} \leq \min \left\{(10 \times M S R),\left(P_{D G i, t}^{\max }-P_{D G i, t}\right)\right\}
$$

Where MSR is power generation increase rate in 1 minute.

- Interruptible loads limits:

$$
0 \leq\left(A S_{\text {curtk }, t}\right) \times I_{i, t} \leq P_{\text {curtk }, t}^{\max } .
$$

Constraint related to the intra-network of micro-grid is as follow:

- Balance between active and reactive power consumption and generation:

$$
\begin{gathered}
P_{i, t}\left(V_{t}, \theta_{t}\right)-P_{g_{i, t}}+P_{d_{i, t}}=0 \\
Q_{i, t}\left(V_{t}, \theta_{t}\right)-Q_{g_{i, t}}+Q_{d_{i, t}}=0 .
\end{gathered}
$$

Where $\mathrm{d}$ is consumption in node, $\mathrm{g}$ is generation in node and I refers to the node.

- Node voltage limits (ranges):

$$
V_{i}^{\min } \leq V_{i, t} \leq V_{i}^{\max }
$$

- Exchanged power limit

$$
\left|E_{t}+A S_{t}\right| \leq E_{\text {exch }}^{\max } .
$$

Where max is maximum Exchanged power with the main grid. 


\section{PROPOSED METHOD}

Uncertainty factors like renewable DG unit's power output, load, price of energy and ancillary services markets and reliability (fail) of production units and lines, can affect the optimal performance of micro-grid. Traditional methods, which considered only one scenario for micro-grid can be invalid and can affect micro-grid's expected total profit from participating in day ahead electricity market. Therefore, a reasonable bidding strategy must consider these uncertainties. In this article, the uncertainties of the output power of renewable units, load and fluctuations in the market prices are taken in to account for the presence of the micro-grid in energy and spinning reserve day ahead markets. In following sections, the proposed method will be presented.

\subsection{Forecasting of uncertainty factors}

For the presence of micro-grid in electricity market, it is essential that central controller sends its bids to the market operator. The submitted bids to the electricity market by central controller, will determine the micro-grid profit. Therefore accurate estimation of uncertainty factors, including wind speed, solar radiation, load and price fluctuations will improve microgrid profit.

Various studies have developed various methods for more accurate prediction of these factors. Among these neural network, models based on time series, a variety of innovative methods and etc, can be mentioned. In this paper, for forecasting each factor of uncertainties, an adaptive fuzzy neural inference system (ANFIS) (which adapted from [8]) was used. This algorithm is used to find the next DM prices based on the knowledge of previous day's data. Firstly, ANFIS is trained and then ANFIS will do predictions with high accuracy.

In this research, for training ANFIS, the information of last 7 days, prices of the same day in the last year, day-type of the target day which is considered zero for the weekend and 1 for the weekdays and weather factor are used as the input of learning machine. For predicting the uncertainty factors, initially a parameterized model for determining relationship between input and output, membership function and rules governing training will be used. Next, input/output data, which can be used for training ANFIS, will be collected. Then ANFIS can be used for Forecasting. Forecasting method structure is shown in Fig. 2. Also Historical data of load, wind speed, solar irradiation and market prices of Spain are available in [10] to [13] respectively. 


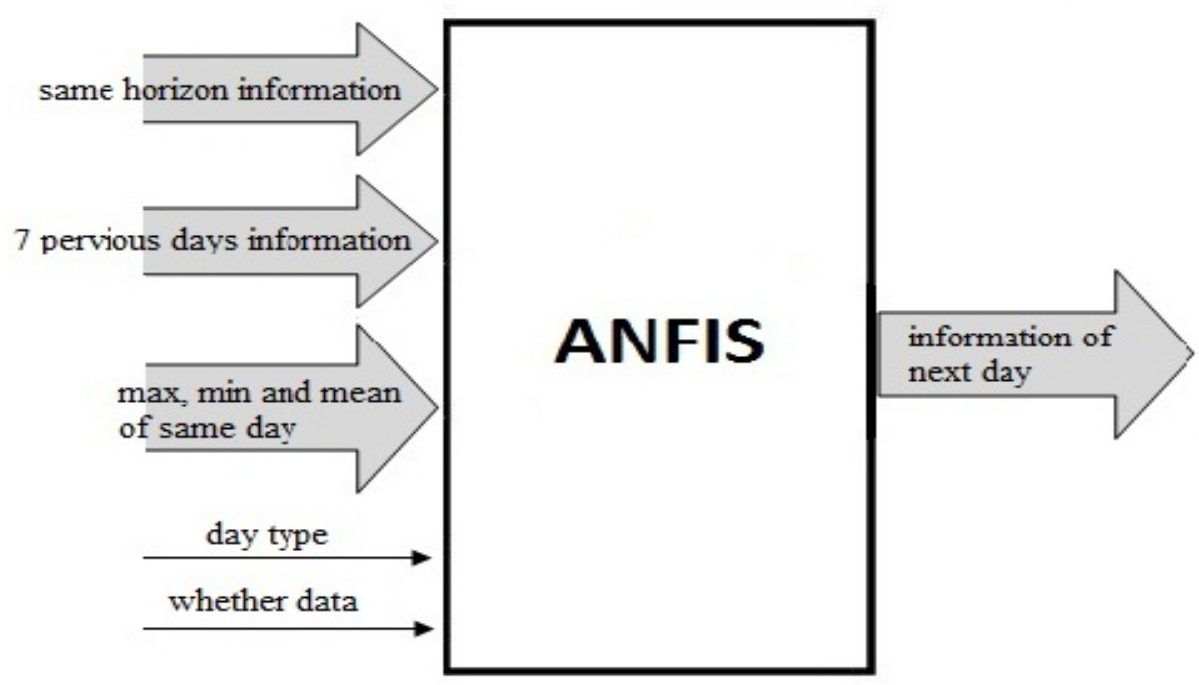

Fig. 2: ANFIS framework

\subsection{Scenario generation}

Due to existing uncertainties, Micro-grid scenarios must be produced. Uncertainties of wind speed, solar irradiation, load and the price fluctuations cause various scenarios. For optimal performance, a wide range of scenarios must be modeled. Latin Hypercube Sampling (LHS) is a group sampling in which a full coverage of the range of variable with sufficient accuracy will be modeled, so the LHS method was used for the scenario production of the micro-grid. The process is as the following.

Step 1: first, based on the method described in the previous section, for one month before the day under study necessary predictions will be done. Then with respect to errors in this 30-day predictions, forecasts for the under study day will be provided.

Step2: According to the forecast errors of previous step, the predictions is done for study day.

Step 3: Calculation of the output power of renewable units with respect to the wind speed, irradiation and the power transmission curves.

Step 4: obtaining a cumulative distribution function of each of the variables and dividing it into $\mathrm{N}$ equal parts.

Step 5: choosing a number randomly in any distance and Cumulative probability distance $\mathrm{i}$ is as following. 


$$
\operatorname{prob}_{i}=\frac{1}{N} r_{u}+\frac{i-1}{N}
$$

Where $r_{u}$ is a random number between zero and one.

Step 6: transforming selected probability values (prob) into the numerical values of variables $\left(x_{e}\right)$ by reverse cumulative distribution function $\left(F^{-1}\right)$.

$$
x_{e}=F^{-1}(\text { prob })
$$

\subsection{Scenario reduction}

There are lot of Scenarios that are generated by LHS for micro-grid and this leads to increase in time and cost of calculations. Therefore with sufficient accuracy, the number of scenarios must be reduced. There are various methods to reduce the number of scenario that are reported in [9]. According to relative distance between scenarios, the backward scenario reduction technique deletes a scenario in each iteration and can bring about good accuracy, so in this paper, this method is used for reduction of scenarios as following:

Step 1: Based on the scenarios generated by LHS, the probability of each scenario is equal to and the desired number of scenarios is $n^{*}$ which in the beginning the number of reduced scenarios $n^{*}=\mathrm{N}$.

Step 2: Calculation of the Kantorovich distance for each pair of scenarios.

$$
\begin{aligned}
& \mathbf{K D}\left(S_{i}, S_{j}\right)=\left|\mathbf{B}_{i, t}-\mathbf{B}_{j, t}\right| . \\
& \mathbf{B}_{i, t}=\left(\mathbf{E} p_{i, t}+\mathbf{R} p_{i, t}\right) \times\left(\mathbf{L o a d}_{i, t}-\mathbf{W} \mathbf{P}_{i, t}-\mathbf{P} \mathbf{V}_{i, t}\right) .
\end{aligned}
$$

Where $\mathbf{E} p:$ is the price of energy markets, $\mathbf{R} p:$ the market price of spinning reserve, LOAD: load of micro-grid,WP: wind power and PV is PV power of micro-grid.

Step3: selecting the scenario that has the minimum Kantorovich distance with scenario i:

$$
\mathbf{K D}\left(S_{i}, S_{k}\right)=\min \left(\mathbf{K D}\left(S_{i}, S_{j}\right)\right) \quad 1 \leq j \leq n^{*}
$$

Then the probability of scenario will be calculated.

$$
\operatorname{PKD}\left(S_{i}, S_{k}\right)=\mathbf{K D}\left(S_{i}, S_{k}\right) \times \mathbf{P}_{i} .
$$

Where $P_{i}$ is the probability of scenario $\mathrm{i}$.

Step 4: Repeating step 3 for all scenarios and calculating: 


$$
\operatorname{PKD}\left(S_{i}, S_{k}\right)=\min \left(\mathbf{P K D}\left(S_{i}, S_{k}\right)\right) 1 \leq k \leq n^{*} .
$$

Step 5: between selected scenarios with regard to the event probability of chosen scenarios, one of the scenarios will be deleted and its probability will be added to the nearest possible scenario and the number of scenarios will be updated.

Step 6: the steps2 to5 will be repeated up to reaching to the desired number of scenario.

\subsection{Objective function optimization}

Based on the chosen scenarios from scenario reduction section, each scenario is considered as a deterministic scenario and objective function is optimized by genetic algorithm with the aim of maximizing profit of micro-grid from participating in the electricity markets. At the end, based on the (event) probability of each scenario, micro-grid total expected profit is calculated.

\section{CASE STUDY}

To evaluate the proposed method, different simulations and analyses on a sample microgrid are performed (as shown in Figure 3). This micro-grid consists of three commercial, industrial and residential feeders. Also micro-grid has three $15 \mathrm{~kW}$ wind turbines, $10 \mathrm{~kW}$ photovoltaic unit, $80 \mathrm{~kW}$ fuel cell capacity and $80 \mathrm{~kW}$ system micro-turbine. Detailed information about dispatchable DG units is given in table 1. All DG work at unity power factor. In the following sections two scenarios will examine the effectiveness of the proposed method.

\subsection{Scenario production for micro-grid}

As mentioned in the previous section for considering the uncertainties of renewable output power, load and price fluctuations, multiple scenarios for covering a wide range of possibilities must be produced. To this end, first the predictions of the factors of uncertainty for 30 days of the second month of 2013 will be predicted. Then based on these predictions totally 300 scenarios will be generated for sixty-second days of 2013. Finally these 300 Scenarios will be reduced to 10 scenarios and will be applied to micro-grid. In the following sections, presence of micro-grid in the energy and spinning reserve markets will be examined. 


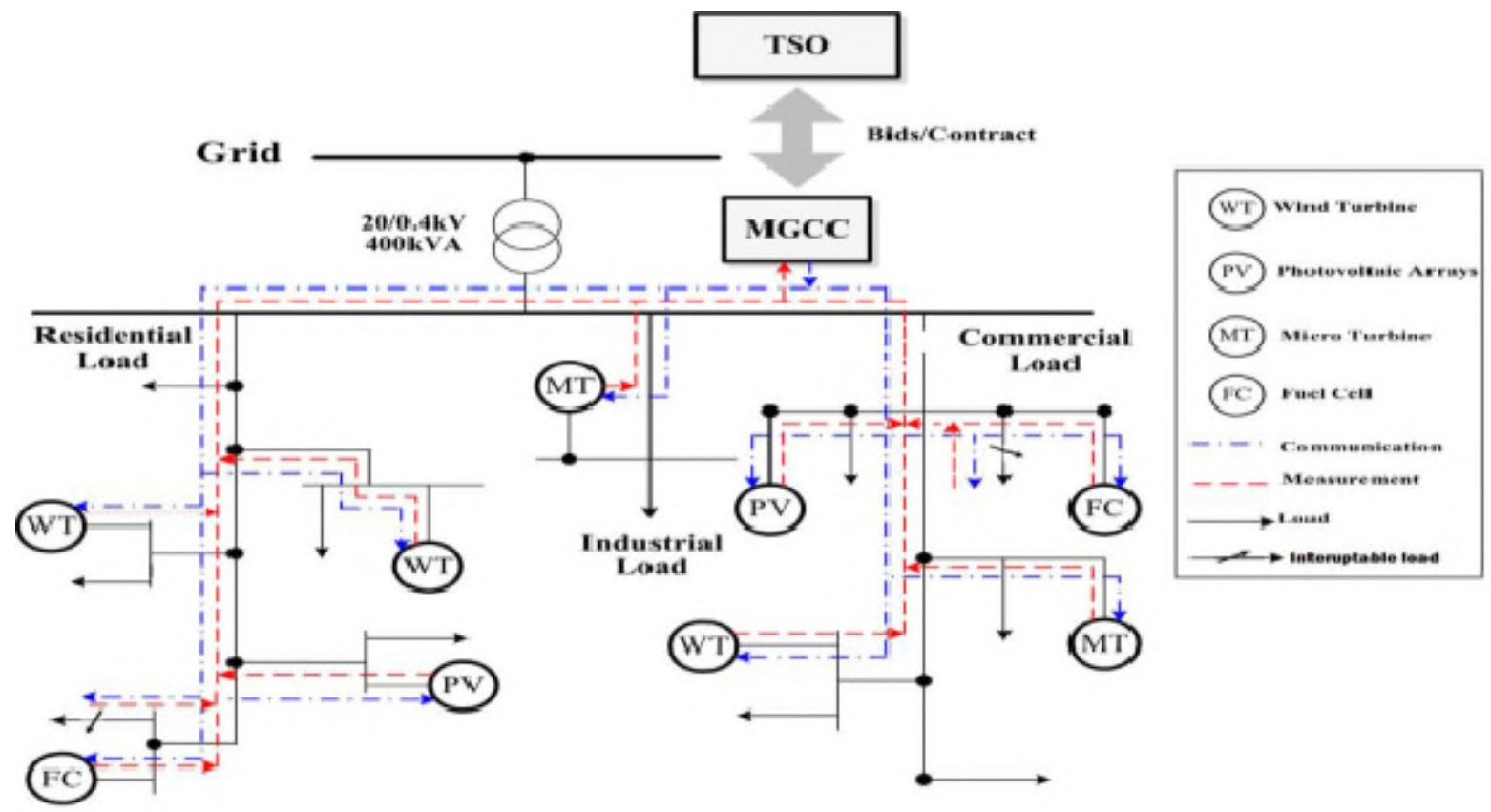

Fig. 3 : Case study [10]

Table 1 : Dispatchable DG information

\begin{tabular}{ccccccc}
\hline DG unit & $\begin{array}{c}\text { Min } \\
\text { power } \\
(\mathrm{kW})\end{array}$ & $\begin{array}{c}\text { Max } \\
\text { power } \\
(\mathrm{kW})\end{array}$ & $\begin{array}{c}a_{i} \\
(\text { Cent/kWh })\end{array}$ & $\begin{array}{c}b_{i} \\
\text { Cent/h })\end{array}$ & $\begin{array}{c}\text { Startup } \\
\text { cost }\end{array}$ & $\begin{array}{c}\text { Shut } \\
\text { down } \\
\text { cost }\end{array}$ \\
\hline \hline DG1(FC) & 3 & 40 & 2.84 & 155.18 & 9 & 8 \\
DG2(MT) & 3 & 40 & 2.84 & 155.18 & 9 & 8 \\
DG3(MT) & 3 & 40 & 4.47 & 85.06 & 16 & 9 \\
DG4(MT) & 3 & 40 & 4.47 & 85.06 & 16 & 9 \\
\hline
\end{tabular}

\subsection{Participation in energy market}

First, we assume issue for the sixty-second day of year without any uncertainty. Information of this day and the results of the simulation are shown in Figures 4 and 5 respectively. As it is shown, between the 3A.M to 7 A.M the load of system is in its minimum value and micro-grid has found an opportunity to sell energy to the main network. At 10A.m. to $12 \mathrm{~A} . \mathrm{m}$. , based on the energy price and retail rate, it has been decided to decrease the microgrid local production and required power purchased from the grid. And then with the rise of prices in electricity market, the internal market production has increased. The expected total profit of micro-grid in this case is -19174 cents. It should be noted that in due to low domestic 

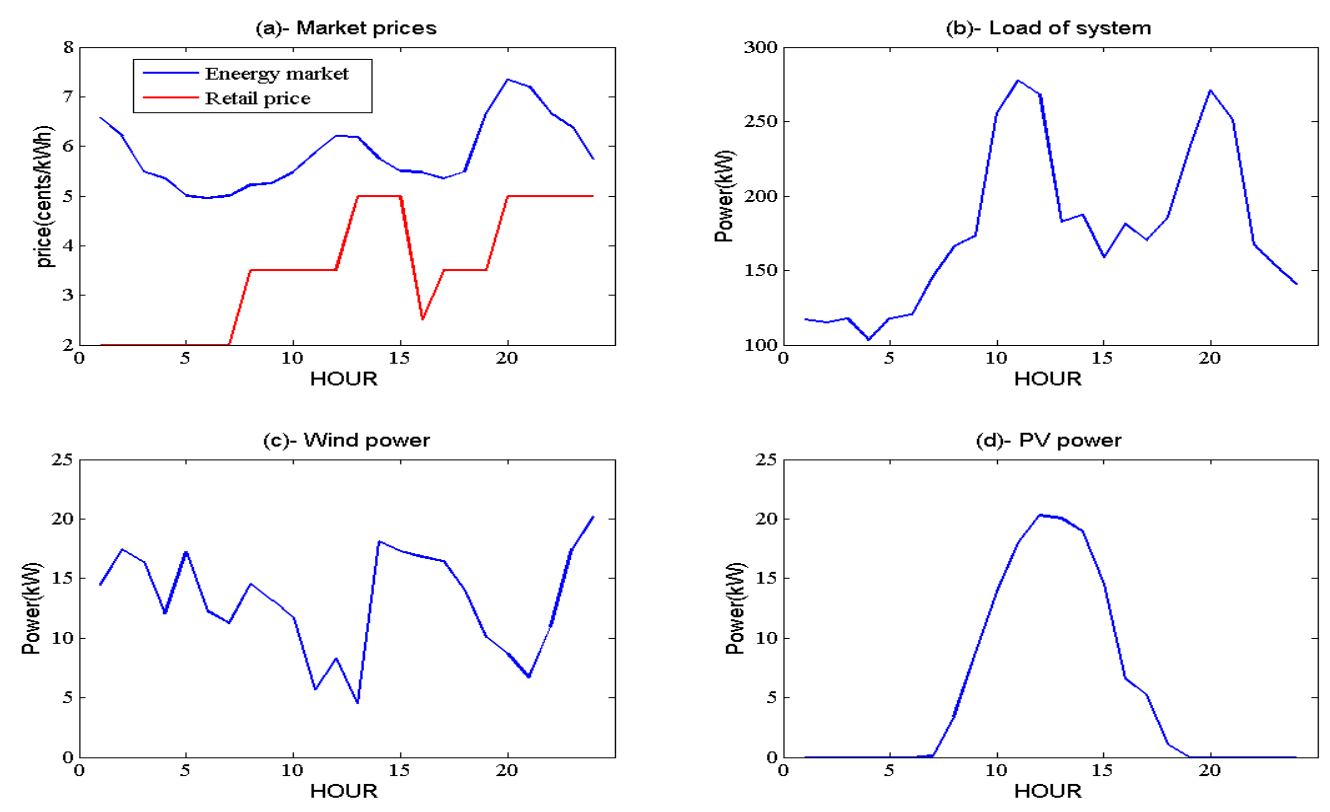

Fig. 4 : Information of sixty-second day
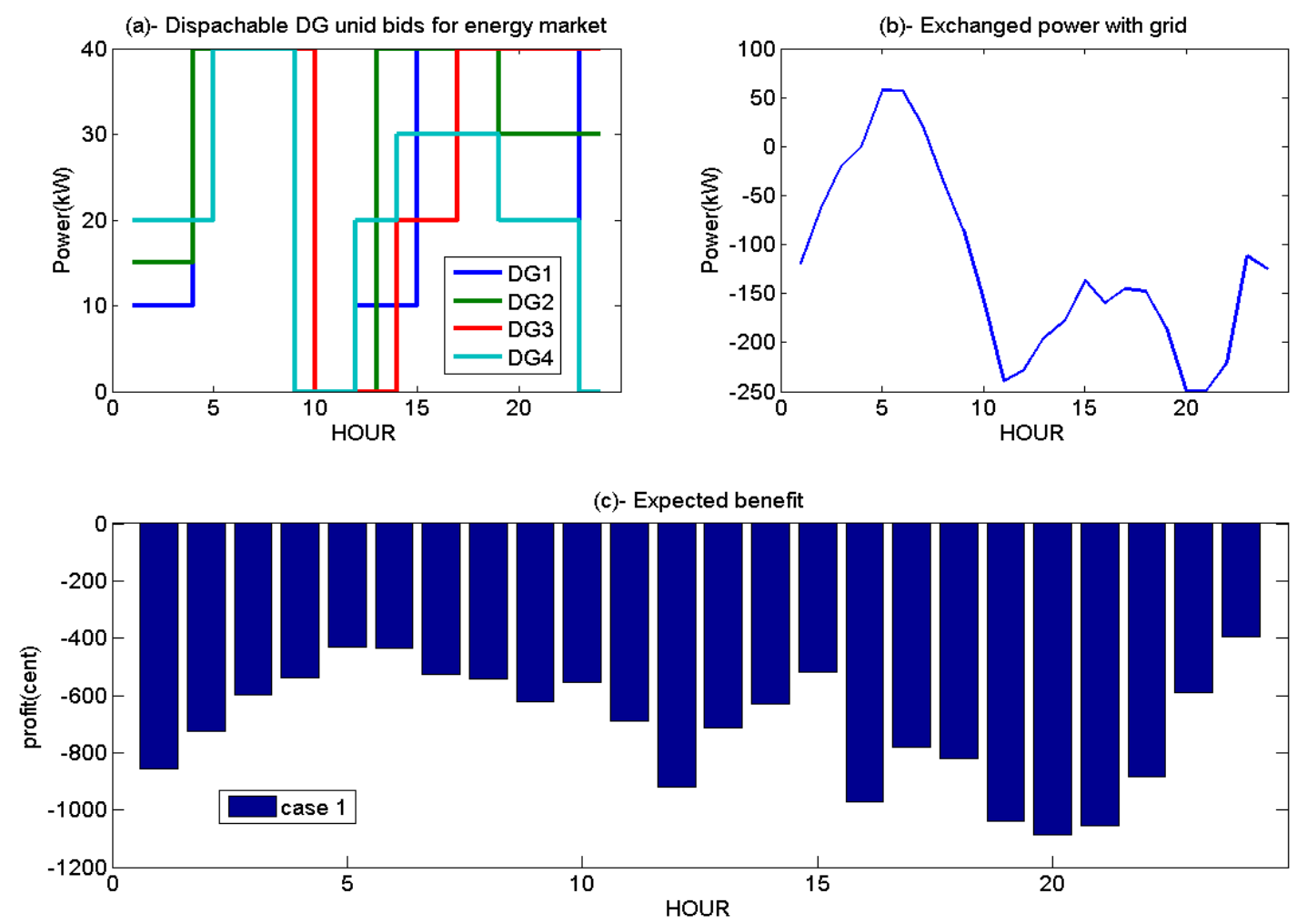

Fig. 5 : A- bids od dispatchable dg units for participating in energy market, b- exchanged power with main grid, c- expected profit 
production capacity of the micro-grid, most of the time micro-grid has to buy power from the upstream network.

Then the proposed method is applied to the micro-grid and it is observed that the expected profit of micro-grid has increased to -13685 cents. For further investigation of the effectiveness of the proposed method, the method proposed in reference [7] (where uncertainty in the market price is lack of consideration) will be applied to the micro-grid. The expected profit of micro-grid in this case is -15463 cents. The result indicates that the influence of price fluctuations in profitability of micro-grid. This is shown in Figure 6 where a comparison between the proposed method and other methods is done.

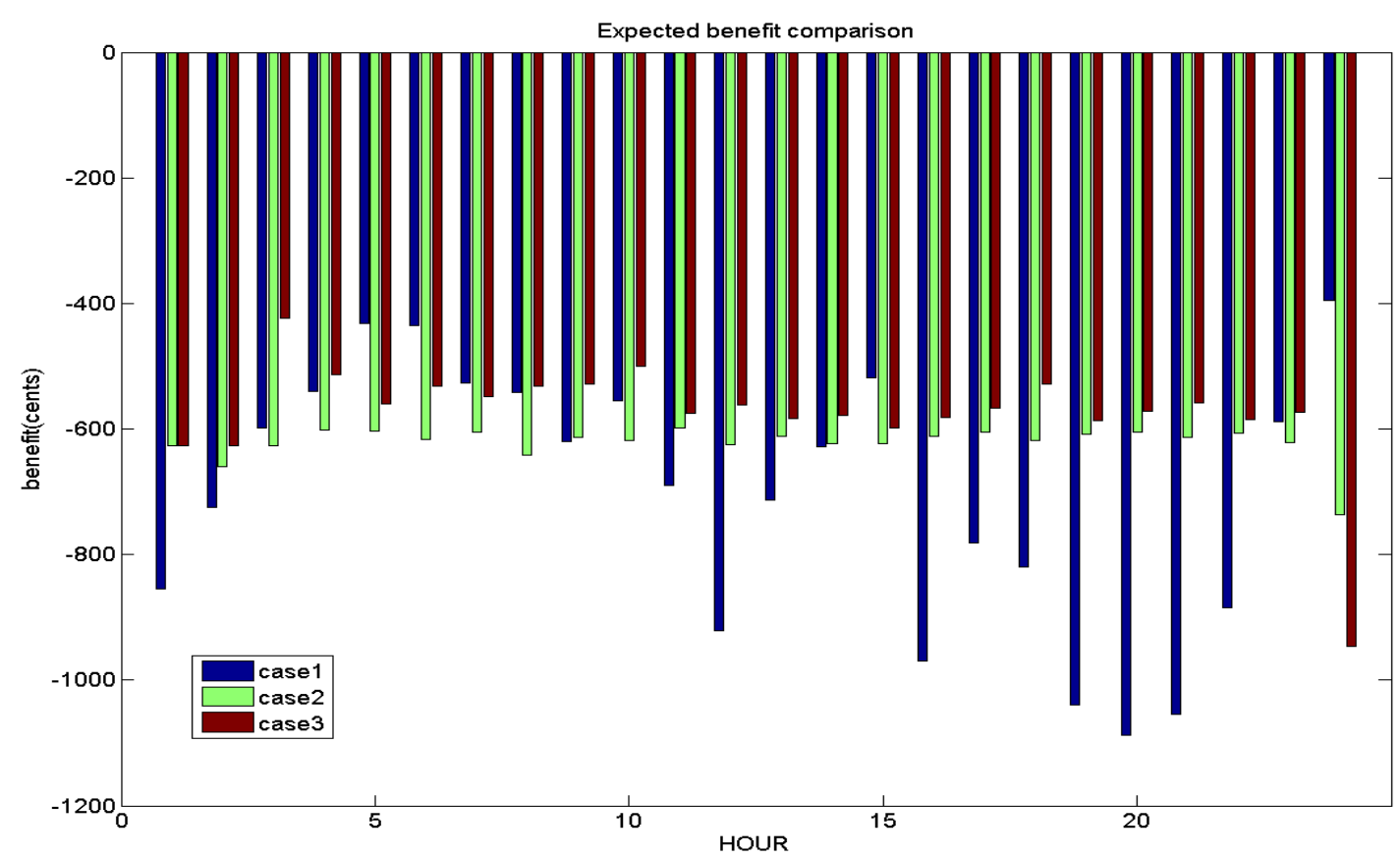

Fig. 6: Profit comparsion between three mode in scenario 1

\subsection{Participation in energy and spinning reserve markets}

In this case, it is assumed that the dispatchable distributed generation units consider $25 \%$ of their capacity for participating in spinning reserve market. The micro-grid can reduce its interruptible loads up to $20 \mathrm{~kW}$ between 8 and 24 P.m. The cost of load curtailment is 5 cent $/ \mathrm{kWh}$. 
As in the previous case, in three different modes, (regardless of the uncertainty, regardless of price uncertainty and the proposed method) the proposed method will be evaluated. The simulation results show that the proposed method compared to the two other modes (regardless uncertainty, regardless price uncertainty) is 3358 and 1149 cents more profitable for micro-grid respectively. Results of Comparison of three methods are shown in Figure 7. It can be also seen that the presence in spinning reserve market is profitable. For further evaluation of the proposed method, we will examine participation of micro-grid in electricity markets for 7 more days. The results are illustrated in Figure 8 and indicate the importance of electricity market price fluctuations in the profitability of micro-grid and efficiency of the proposed method.

\section{CONCLUSION}

With the regulation change in the electricity industry, the issue of interaction of microgrids with traditional distribution systems as well as maximization of potential benefits of micro-grids in the competitive environments for reception and expansion of distributed productions, is of high importance. Besides that due to the impact of uncertainties on the optimization problem, in this paper, a new method for biding strategy, which consider uncertainties of power market, is suggested. In this proposed method the ANFIS method was used for predicting uncertainty factor, LHS method was used for generation of scenarios and backward scenario reduction method was used for reducing the calculation time. This method is tested on a sampled micro-grid in two different scenarios. The simulation result revealed the efficiency of the method.

With respect to the influence of uncertainties on the profitability of micro-grid, it is required that in the future studies the uncertainties of unit status be considered in the issue. Furthermore in this research, it is assumed that micro-grid is price taker. With the increasing influence of disturbed generation in power systems, the assumption of effectiveness of microgrids on the price market seems reasonable and should be studied in the further investigations. 


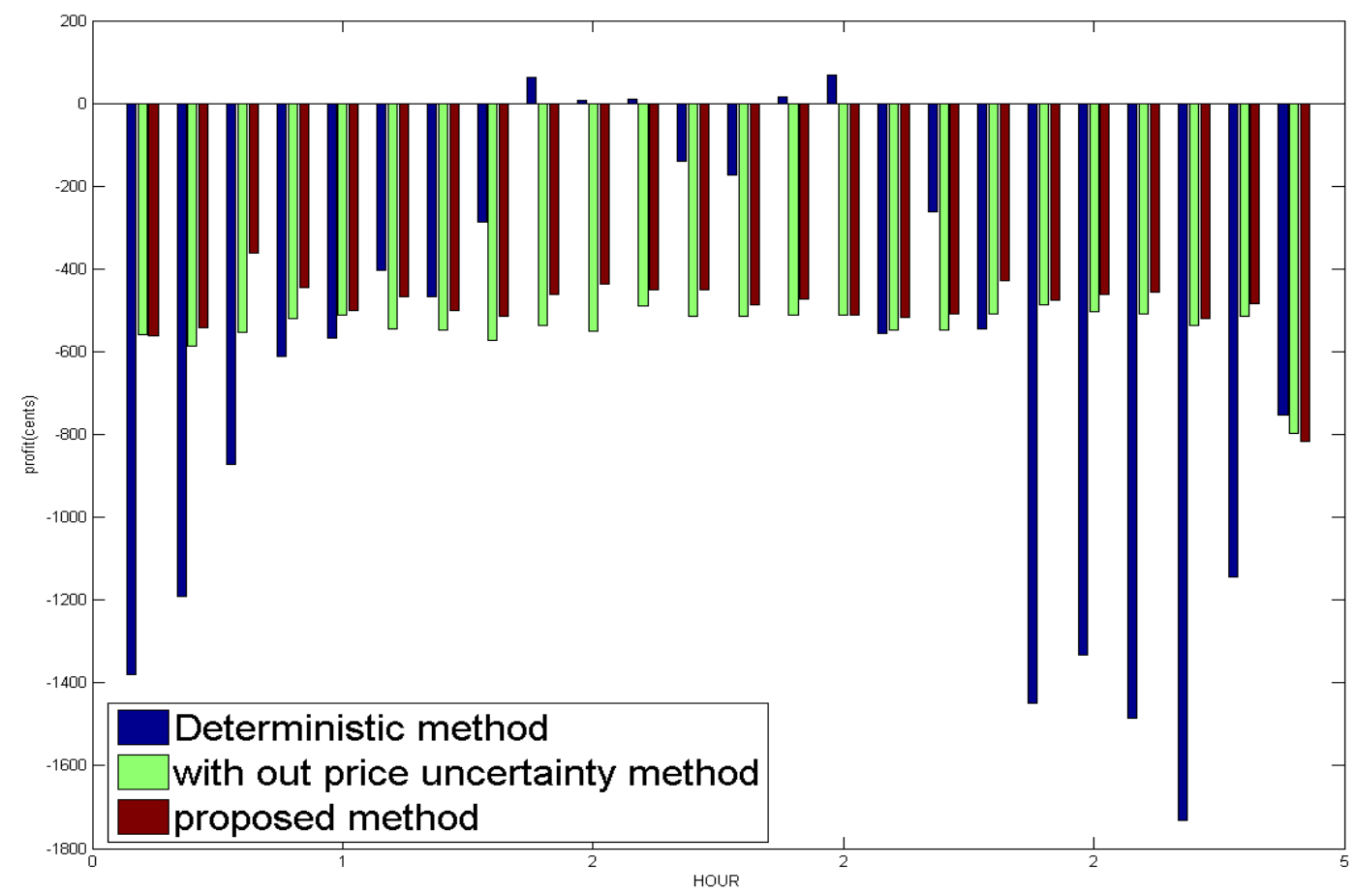

Fig. 7 : Profit comparsion between three mode in scenario 2

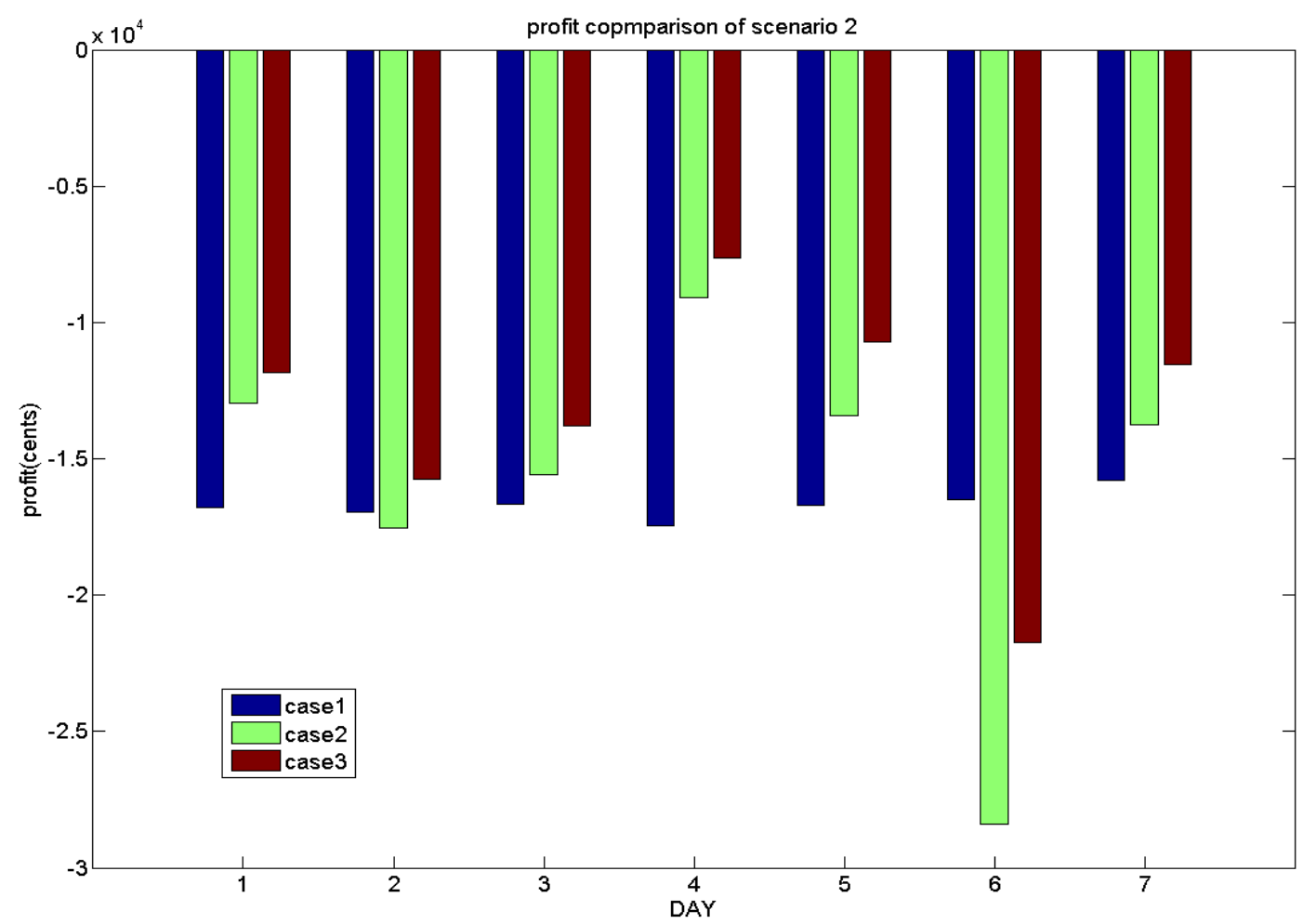

Fig. 10 : Profit comparsion of scenario 2 for 7 days 


\section{REFERENCES}

1. A. C. Rueda-Medina and A. Padilha-Feltrin, Distributed generators as a providers of reactive power support, IEEE Trans. power systems, vol. 28, no. 1, pp.490-502, 2013.

2. E. Hirst and B. Kirby, Electric-power ancillary services, Oak Ridge National Laboratory, pp. 48, 1996.

3. E. Mashhour and S. M. Moghaddas-Tafreshi, Bidding strategy of virtual power plant for participating in energy and spinning reserve markets- Part I: Problem formulation, IEEE Trans. Power Syst., vol. 26, no.2, pp.949-956, 2011.

4. E. Mashhour and S. M. Moghaddas-Tafreshi, A review on operation of micro grids and virtual power plants in the power markets, IEEE 2nd International Conference on Adaptive Science \& Technology, pp. 273-277, 2009.

5. R. Palma-Behnke, J. L. Cerda, and A. Jofre, A distribution company energy acquisition market model with integration of distributed generation and load curtailment options, IEEE Trans. Power Syst., vol. 20, no. 4, pp. 1718-1727, 2005.

6. T. ares et al, Smart grid market using joint energy and ancillary services bids, IEEE Grenoble In PowerTech (POWERTECH), pp 1-6, 2013.

7. L. Shi, Y. Luo and G. Y. Tu, Bidding strategy of microgrid with consideration of uncertainty for participating in power market, Electrical power and energy systems, vol. 59, pp.1-13, 2014.

8. H. Shayeghi and B. Sobhani, Integrated offering strategy for profit enhancement of distributed resources and demand response in microgrids considering system uncertainties, Enegy conversion and management, pp.765-777, 2014.

9. N. M. M. Razali and A. H. Hashim, Backward reduction application for minimizing wind power scenarios in stochastic programming, Proc 4th international power engineering and optimization conf, p. 430-434, 2010.

10. A. G. Tsikalakis and N. D. Hatziargyriou , Centrolized control for optimizing microgrids operation, IEEE Trans. Energy Convers, vol. 23, no. 1, pp. 241-248, 2008.

11. http://www.sotaventogalicia.com/index.php

12. http://www.solargis.com

13. http://www.esios.ree.es/web-publica 